\title{
Qualidade de vida de pacientes hospitalizados com feridas crônicas
}

Kezia Cristina Batista dos Santos ${ }^{1}$, Gabriela Sellen Campos Ribeiro ${ }^{2}$, Adrielly Haiany Coimbra Feitosa ${ }^{3}$, Barbara Regina Souza da Silva ${ }^{4}$,

Tamires Barradas Cavalcante ${ }^{5}$

\section{RESUMO}

Objetivou-se analisar a qualidade de vida de pacientes hospitalizados com feridas crônicas. Estudo analítico, transversal com abordagem quantitativa. A amostra do estudo foi composta por 30 pacientes internados com feridas crônicas em um hospital universitário do nordeste do Brasil, no período de agosto de 2017 a janeiro de 2018. Para coleta de dados utilizou-se um questionário de avaliação mental, um formulário socioeconômico e clínico e a versão brasileira do Freiburg Life Quality Assessment Wound. O domínio com maior interferência na qualidade de vida dos pacientes hospitalizados foi vida social e o de menor interferência, bem-estar psicológico. Foi encontrada associação significativa entre maior idade e mobilidade totalmente limitada com uma pior qualidade de vida dos pacientes internados. Este estudo contribui para o planejamento e implementação de ações de enfermagem com foco nos aspectos biopsicossociais que colaborem para uma melhor qualidade de vida dos pacientes e como subsídio para pesquisas futuras.

Descritores: Qualidade de Vida; Ferimentos e Lesões; Pacientes Internados; Cuidados de Enfermagem.

\footnotetext{
${ }^{1}$ Enfermeira. Programa de Pós-Graduação em Enfermagem da Universidade Federal do Maranhão, nível Mestrado. São Luís, MA, Brasil. E-mail: kezia_cristinabs@hotmail.com.

2 Enfermeira. Professora da Escola Técnica de Bacabeira. Bacabeira, MA, Brasil. E-mail: gabrielasellen@gmail.com.

${ }^{3}$ Enfermeira. São Luís, MA, Brasil. E-mail: adrielly.enf.coimbra@hotmail.com.

${ }^{4}$ Enfermeira. Enfermeira Assistencial do Hospital São Domingos. São Luís, MA, Brasil. E-mail: barbara.rsss@ hotmail.com.

${ }^{5}$ Enfermeira, Mestre em Enfermagem. Discente do Programa de Pós-Graduação em Saúde Coletiva pela Universidade Federal do Maranhão, nível Doutorado. Enfermeira da Unidade de Traumato-Ortopedia e preceptora da Residência Multiprofissional em Saúde do Hospital Universitário do Universidade Federal do Maranhão. São Luís, MA, Brasil. E-mail: tamiresbarradas@gmail.com.
}

Artigo recebido: 23/07/2018.

Artigo aprovado: 21/11/2018.

Artigo publicado: 31/12/2018.

\section{Como citar esse artigo:}

Santos KCB, Ribeiro GSC, Feitosa AHC, Silva BRS, Cavalcante TB. Qualidade de vida de pacientes hospitalizados com feridas crônicas. Rev. Eletr. Enf. [Internet]. 2018 [acesso em: ];20:v20a49. Disponível em: 


\section{INTRODUÇÃO}

Feridas crônicas são definidas como rupturas na pele com período de duração superior a seis semanas, que não seguem o processo cicatricial normal e que podem persistir apesar da implementação de cuidados adequados $^{(1)}$.

No Brasil, apesar de registros escassos, as feridas crônicas constituem sério problema de saúde pública, devido ao impacto psicológico, social e econômico gerados ao paciente, associado aos elevados e crescentes custos para o sistema de saúde ${ }^{(2)}$.

Dentre as feridas crônicas com maior prevalência no Brasil, destaca-se a lesão por pressão (LP), afetando aproximadamente $25,9 \%$ dos pacientes hospitalizados, principalmente os idosos ${ }^{(3)}$. Em segundo lugar, úlcera diabética (UD), com prevalência de 62,5\% entre 42 pacientes hospitalizados ${ }^{(4)}$. E as feridas operatórias complicadas (FOC), com incidência de $6 \%$ em ambiente hospitalar, como identificado em um estudo no Brasil ${ }^{(5)}$, enquanto nos EUA, a prevalência foi de apenas $2 \%$ a $5 \%{ }^{(6)}$.

Estudos comprovam que pacientes com feridas crônicas apresentam qualidade de vida (QV) pior em relação aos que não são afetados, devido à dor, dificuldade de mobilidade, frustração, ansiedade, depressão, isolamento social, e, frequentemente, alteração da imagem corporal, acarretando mudanças no estilo de vida, piora na autoestima e em sua capacidade funcional, impossibilitando-os, muitas vezes, de exercer suas atividades de vida diária e laborais ${ }^{(7-8)}$.

O crescente número de pessoas com feridas crônicas contribui para o aumento dos gastos públicos em saúde, além de interferir diretamente na QV da população. Para evitar que isso ocorra, a equipe multiprofissional deve proporcionar assistência global buscando atender às necessidades biopsicossociais para melhorar as condições de vida destes indivíduos ${ }^{(2)}$. Neste contexto, a Enfermagem é extremamente importante, pois na prevenção e tratamento de feridas o enfermeiro é o profissional que acompanha a evolução da lesão, fornece orientações e executa o curativo(9).

Entretanto, a forma como os pacientes lidam com o problema e percebem o apoio familiar são desafios enfrentados pelos enfermeiros. Logo, a avaliação da QV é um mecanismo importante para mensurar os resultados das intervenções de saúde, assim como direcionar a elaboração e estabelecimento de metas terapêuticas com foco nos aspectos biopsicossociais individuais dos pacientes para prevenção do problema ${ }^{(10)}$.

A dificuldade de avaliar a QV de pacientes com feridas crônicas é um desafio para os profissionais de saúde devido às necessidades específicas de saúde dessa população ${ }^{(10)}$. No tratamento de feridas, esta dificuldade é minimizada devido à disponibilidade de instrumentos específicos de avaliação de $Q V$, como o $F L Q A-W k$, que recentemente foi traduzido para português brasileiro ${ }^{(11)}$. o FLQA-Wk é a versão abreviada do Freiburg Life Quality Assessment-Wound (FLQA-W) ${ }^{(12)}$ validado em 2010 e utilizado em diversas pesquisas em vários países ${ }^{(13-16)}$. No Brasil, ainda não existem estudos que utilizaram o FLQA-Wk para avaliar a QV de pacientes com feridas crônicas ${ }^{(17)}$.

Pacientes com feridas crônicas são atendidos nos diferentes pontos da rede de atenção à saúde. A maioria dos estudos são realizados com pacientes atendidos em unidades básicas de saúde e ambulatórios especializados. Os estudos realizados em ambiente nosocomial são voltados para feridas crônicas de etiologia específica, tais como: LP e UD, entretanto, pouco se aborda sobre os demais tipos. A internação é um momento oportuno para 
intervenções com o foco na QV, seja por meio de orientações ou pelo uso de outras tecnologias para tratamento de feridas características deste ambiente.

Diante disto, tendo em vista a diversidade de feridas crônicas, a necessidade de avaliar a QV de pacientes hospitalizados com feridas crônicas e disponibilidade de instrumentos específicos com nova perspectiva sobre a avaliação da QV, objetivou-se analisar a QV de pacientes hospitalizados com feridas crônicas utilizando a versão brasileira do instrumento Freiburg Life Quality Assessment Wound (FLQA-Wk)(11).

\section{MÉTODOS}

Estudo analítico, transversal com abordagem quantitativa desenvolvido no Hospital Universitário da Universidade Federal do Maranhão (HUUFMA) Unidade Presidente Dutra, no município de São Luís do Maranhão, Brasil, entre os meses de agosto de 2017 a janeiro de 2018. O hospital é considerado referência do estado e responsável pela assistência a pacientes eletivos e de urgência clínica/cirúrgica e traumatológica, com serviço de estomaterapia coordenado por uma enfermeira estomaterapeuta.

A população correspondeu a 71 pacientes com feridas crônicas internados nas Unidades de Internação Clínica e Cirúrgica do referido hospital. Foram incluídos na pesquisa os pacientes com idade $\geq 18$ anos, presença de uma ou mais feridas crônicas, internados nas referidas unidades de internação e que atingiram escore $\geq 07$ pontos no Questionário de Avaliação Mental validado para o português ${ }^{(18)}$. O questionário de Avaliação Mental utilizado no estudo é de domínio público, consiste em 10 perguntas, as quais avaliam se o indivíduo sofre ou não de uma síndrome mental orgânica. O entrevistado deve acertar, no mínimo, sete das dez perguntas, totalizando o mínimo de sete pontos ${ }^{(18)}$.

Foram excluídos os pacientes com incapacidade de compreensão e comunicação verbal efetiva, pacientes com feridas oriundas de queimaduras extensas, neoplásicas malignas ou portadores da Síndrome de Imunodeficiência Adquirida (SIDA). Após aplicação dos critérios de inclusão e exclusão a amostra de conveniência foi constituída de 30 pacientes, coletados conforme possibilidade de aplicação dos instrumentos e realização de exame físico da pele para avaliação da ferida em tempo hábil.

A coleta dos dados foi realizada pelas pesquisadoras por meio de entrevista individual nos turnos matutino e vespertino, duas vezes na semana, sendo dividida em duas etapas: busca ativa nas unidades de internação da instituição a partir da lista de pacientes internados fornecida pelo setor de tecnologia e informática, aplicação do Questionário de Avaliação Mental(18), formulário semiestruturado referente às variáveis socioeconômicas e clínicas, e aplicação do FLQA-W $k^{(11)}$; exame físico da pele para identificação e avaliação das feridas crônicas. Os instrumentos de coleta de dados foram aplicados individualmente, no leito, após explanação sobre a pesquisa e assinatura do Termo de Consentimento Livre e Esclarecido, em duas vias.

As variáveis socioeconômicas e clínicas utilizadas no estudo foram: sexo, idade, cor da pele, procedência, renda familiar, escolaridade, tempo de internação, mobilidade, tipo de ferida, número e área da ferida, sinais de cicatrização e sinais de infecção.

O exame físico da pele e avaliação das feridas crônicas foram realizados de forma observacional durante a realização do curativo. A avaliação da medida da ferida foi realizada de forma bidimensional com mensuração das 
dimensões comprimento e largura e auxílio de uma régua de papel descartável individual para cada paciente, com

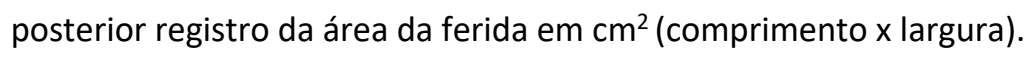

No caso de pacientes com mais de uma ferida, a área total foi obtida a partir da soma das áreas de todas as feridas do paciente. Para avaliação dos sinais de cicatrização da ferida classificou-se quanto a presença ou não de sinais de cicatrização (presença de tecido de granulação). Os sinais de infecção foram avaliados de acordo com a presença das características: eritema, edema e presença de exsudato purulento, simultaneamente. A mobilidade foi avaliada por meio das categorias: não apresenta limitações, levemente limitado, bastante limitado e totalmente limitado.

O Freiburg Life Quality Assessment-Wound (FLQA-W) é um instrumento utilizado mundialmente, validado em 2010 e recentemente adaptado para a língua portuguesa do Brasil(11). O FLQA-Wk(11) é a versão abreviada do FLQA-W ${ }^{(12)}$ e tem como objetivo mensurar a QV de pessoas com feridas crônicas. É composto por 24 itens, distribuídos em seis domínios: sintomas físicos, vida diária, vida social, bem-estar psicológico, tratamento e satisfação $^{(11)}$. Cada questão é pontuada de acordo com a resposta do entrevistado variando de um a cinco. Os domínios são calculados pela média aritmética de cada resposta e o escore total é computado pela média dos valores de cada domínio. O escore varia de um (melhor QV) a cinco (pior QV), exceto no domínio satisfação que deve ser recodificado. Quanto maior o valor do escore, pior a $\mathrm{QV}^{(11-12)}$.

O instrumento FLQA-Wk também apresenta três escalas visuais analógicas, graduadas de zero (muito ruim) a dez (muito bom) que avaliam a QV, saúde em geral e condições da ferida na última semana. $O$ total da escala visual analógica do FLQA-Wk é resultado da média entre as três escalas citadas. Essas escalas auxiliam no controle de valores dos domínios, ou seja, comparam-se os seus valores com o escore total do instrumento ${ }^{(11-12)}$.

Os dados coletados foram inseridos em um banco de dados na planilha do programa Microsoft Excel 2013 com dupla digitação. Logo após, foram exportados e analisados usando recursos da estatística descritiva, utilizando o software Statistical Package for the Social Sciences (versão 20.0). Na análise univariada, foram calculadas média e desvio padrão para as variáveis contínuas e frequência relativa e absoluta para as variáveis categóricas. Na análise bivariada, foi utilizado o teste Shapiro-Wilk para verificação da normalidade e para verificar a associação entre as variáveis do estudo e a QV foram utilizados Teste T Student, Anova e correlação de Pearson e correlação de Spearman considerando o nível de significância de $5 \%(p<0,05)$.

Os aspectos éticos foram respeitados, conforme Diretrizes e Normas Regulamentadoras de Pesquisas Envolvendo Seres Humanos e Resolução do Conselho Nacional de Saúde (CNS) no 466/2012, de forma que o estudo foi submetido e aprovado pelo Comitê de Ética em Pesquisa do Hospital Universitário da Universidade Federal do Maranhão, sob parecer consubstanciado № 2.135.308.

\section{RESULTADOS}

Participaram do estudo 30 pacientes, $70 \%$ do sexo masculino, com idade entre 19 e 77 anos com média de 47,1 (DP $\pm 15,66$ ) anos, 56,7\% de raça parda, 43,3\% apresentaram ensino fundamental incompleto e $40 \%$ renda familiar entre 1-2 salários mínimos. As características das feridas crônicas são mostradas na Tabela 1. 
Tabela 1: Características das feridas crônicas de 30 pacientes hospitalizados. São Luís, MA, Brasil, $2017-2018$.

\begin{tabular}{|c|c|c|c|c|}
\hline Variáveis & n (\%) & Min-Max & Média (DP) & Mediana (AI) \\
\hline Número de feridas & 48 & 01/dez & $1,60( \pm 2,01)$ & \\
\hline Tempo das feridas (mês) & 30 & $1,47-96$ & $6,77( \pm 17,35)$ & $2,47( \pm 2,00)$ \\
\hline Área da ferida $\left(\mathrm{cm}^{2}\right)$ & 30 & $3,00-635,82$ & $62,69( \pm 122,67)$ & $19,75( \pm 41,75)$ \\
\hline Tipo de Ferida & $48^{*}$ & & & \\
\hline Ferida operatória complicada & $12(25,0)$ & & & \\
\hline Lesões esclerodérmicas & $12(25,0)$ & & & \\
\hline Lesão por pressão & $6(12,5)$ & & & \\
\hline Pé diabético & $5(10,4)$ & & & \\
\hline Úlcera vasculogênica & $2(4,2)$ & & & \\
\hline Outros* & $11(22,9)$ & & & \\
\hline \multicolumn{5}{|l|}{ Sinais de cicatrização } \\
\hline Presente & $19(63,3)$ & & & \\
\hline Ausente & $11(36,7)$ & & & \\
\hline \multicolumn{5}{|l|}{ Sinais de infecção } \\
\hline Presente & $23(76,7)$ & & & \\
\hline Ausente & $7(23,3)$ & & & \\
\hline
\end{tabular}

Os dados de QV mostraram que o FLQA-Wk obteve pontuação média de 3,15 (DP $\pm 0,63)$, numa escala que varia de um a cinco sendo que, quanto maior o valor do escore, maior a interferência na QV. Quanto aos escores dos domínios, o domínio bem-estar psicológico obteve menor pontuação 2,15 $(\mathrm{DP} \pm 0,90)$ e o domínio vida social maior pontuação 3,90 (DP $\pm 1,10)$. O valor total da escala visual analógica do FLQA-Wk apresentou média de 5,98 $(\mathrm{DP} \pm 2,05)$, resultado da média das três escalas visuais analógicas. (Tabela 2).

Tabela 2: Escores médios dos domínios, qualidade de vida total e Escalas do FLQA-Wk avaliados em 30 pacientes de feridas crônicas hospitalizados. São Luís, MA, Brasil, 2017-2018.

\begin{tabular}{|c|c|c|c|c|}
\hline Domínios/Escalas & Média & Desvio Padrão & Min & Max \\
\hline Sintomas Físicos & 2,93 & 0,74 & 1,4 & 4,2 \\
\hline Vida Diária & 3,62 & 0,98 & 1,8 & 5 \\
\hline Vida Social & 3,9 & 1,1 & 1,67 & 5 \\
\hline Bem-Estar Psicológico & 2,15 & 0,9 & 1 & 4,5 \\
\hline Tratamento & 3,74 & 0,69 & 2,5 & 5 \\
\hline Satisfação & 2,58 & 0,92 & 1 & 4,33 \\
\hline Total Domínios FLQA-Wk & 3,15 & 0,63 & 1,83 & 4,24 \\
\hline Escala Saúde Geral & 6,53 & 2,35 & 0 & 10 \\
\hline Escala Satisfação com a Ferida & 5,33 & 2,77 & 0 & 10 \\
\hline Escala Qualidade de Vida Geral & 6,06 & 2,75 & 0 & 10 \\
\hline Total Escalas FLQA-Wk & 5,98 & 2,05 & 1 & 9,67 \\
\hline
\end{tabular}

A Tabela 3 apresenta o resultado da associação entre os domínios do FLQA-Wk e as variáveis do estudo. Foi encontrada associação significativa entre mobilidade e os domínios vida diária, tratamento e escore total do FLQAWk e a variável sinais de infecção com o domínio sintomas físicos.

O coeficiente de correlação de Pearson demonstrou correlação positiva estatisticamente significante entre a idade e os domínios Vida Diária $r=0,37(p=0,04)$, Vida Social $r=0,42(p=0,02)$ e escore total do FLQA-Wk $r=0,47$ $(p=0,008)$ indicando que quanto maior a idade, maior os escores destes domínios, o que significa maior prejuízo na vida diária, social e qualidade de vida dos entrevistados; além disso, houve correlação positiva estatisticamente significante em relação ao domínio Satisfação recodificado $r=0,46(p=0,01)$ indicando que quanto maior a idade 
maior o escore, sendo, portanto, maior a satisfação do indivíduo em relação a sua saúde geral, seu tratamento e a aparência de sua ferida. O coeficiente de correlação de Spearman demonstrou correlação positiva estatisticamente significante entre a área total da ferida e o domínio Vida Diária $\mathrm{r}=0,619(p<0,001)$. Tais resultados apontam que quanto maior a área total da ferida, maior os escores de tais domínios e maior a interferência na vida diária e tratamento dos entrevistados.

Tabela 3: Associação entre os domínios do FLQA-Wk com as variáveis avaliadas em 30 pacientes de feridas crônicas hospitalizados. São Luís, MA, Brasil, 2017-2018.

\begin{tabular}{|c|c|c|c|c|c|c|c|}
\hline \multirow{3}{*}{ Variáveis } & \multicolumn{7}{|c|}{ Domínios } \\
\hline & \multirow{2}{*}{$\begin{array}{l}\text { Sintomas } \\
\text { Físicos }\end{array}$} & \multirow{2}{*}{ Vida Diária } & \multirow{2}{*}{$\begin{array}{l}\text { Vida } \\
\text { Social }\end{array}$} & \multirow{2}{*}{$\begin{array}{l}\text { Bem-Estar } \\
\text { Psicológico }\end{array}$} & \multirow{2}{*}{ Tratamento } & \multirow{2}{*}{ Satisfação } & Total \\
\hline & & & & & & & FLQA-Wk \\
\hline \multirow{2}{*}{ Idade } & \multirow{2}{*}{-} & $(r=0,37)$ & $(r=0,42)$ & \multirow[b]{2}{*}{-} & \multirow[b]{2}{*}{-} & $(r=0,46)$ & \multirow{2}{*}{$(\mathrm{r}=0,47) p=0,008^{* * *}$} \\
\hline & & $p=0,04^{* * *}$ & $p=0,02^{* * *}$ & & & $p=0,010^{* * *}$ & \\
\hline Mobilidade & - & $p=0,001^{* *}$ & - & - & $p=0,036^{* *}$ & - & $p=0,021^{* *}$ \\
\hline Área Total da & \multirow{2}{*}{-} & $(0,619)$ & \multirow{2}{*}{ - } & \multirow{2}{*}{-} & \multirow{2}{*}{-} & \multirow{2}{*}{ - } & \multirow{2}{*}{-} \\
\hline Ferida & & $p<0,001^{* * * *}$ & & & & & \\
\hline $\begin{array}{l}\text { Sinais de } \\
\text { infecção }\end{array}$ & $p=0,04^{*}$ & - & - & - & - & - & - \\
\hline
\end{tabular}

A Tabela 4 apresenta o resultado da associação entre as escalas visuais analógicas (EVA) do FLQA-Wk com as variáveis do estudo por meio do Teste T Student. A variável sexo categoria masculino apresentou associação significativa com a EVA saúde geral e as variáveis mobilidade categoria totalmente limitada e sinais de cicatrização categoria presente com a EVA Satisfação com a Ferida.

Tabela 4: Associação entre as Escalas Visuais Analógicas do FLQA-Wk com as variáveis avaliadas em 30 pacientes de feridas crônicas hospitalizados. São Luís, MA, Brasil, 2017-2018.

\begin{tabular}{|c|c|c|c|c|}
\hline \multirow{3}{*}{ Variáveis } & \multicolumn{4}{|c|}{ Escalas } \\
\hline & \multirow{2}{*}{ Saúde Geral } & \multirow{2}{*}{ Satisfação com a ferida } & \multirow{2}{*}{ Qualidade de vida geral } & Total das Escalas \\
\hline & & & & FLQA-Wk \\
\hline Sexo & $p=0,01^{*}$ & & - & - \\
\hline Mobilidade & - & $p=0,05^{*}$ & - & - \\
\hline Sinais de cicatrização & - & $p=0,05^{*}$ & - & - \\
\hline
\end{tabular}

\section{DISCUSSÃO}

As características dos sujeitos foram similares a publicações nacionais e internacionais. Quanto ao sexo, os resultados deste estudo corroboram com estudo de avaliação da QV de pacientes com feridas crônicas realizado no Brasil(19) e com estudo prospectivo que aplicou o FLQA-W em pacientes adultos com feridas crônicas realizado na Alemanha ${ }^{(16)}$, atendidos ambulatorialmente e hospitalizados ${ }^{(20)}$, porém, diverge de outros resultados encontrados na literatura(13-15). O predomínio do gênero masculino pode estar associado à limitação de acesso aos serviços de saúde, demora na busca por atendimento ou ao fato de não admitirem a necessidade de serem cuidados $^{(21)}$.

Em relação à idade os resultados apontam que a média encontrada foi menor que nos outros estudos realizados com pacientes com feridas crônicas ${ }^{(11-12-15)}$, tal fato pode ser justificado pela amostra ser composta por pacientes com tipos de feridas não necessariamente associadas à maior idade, mas sim à hospitalização. 
Entretanto, estudos apontam que o surgimento das feridas crônicas tem se tornado cada vez mais comum com o envelhecimento da população, aumento da expectativa de vida, e o aparecimento de fatores de risco tais como: tabagismo, obesidade, sedentarismo e doenças crônicas como o diabetes mellitus, que predispõe o surgimento destas enfermidades ${ }^{(6,19)}$.

Quanto à escolaridade, o estudo de tradução do FLQA-W para língua portuguesa brasileira ${ }^{(11)}$ evidenciou maior frequência do ensino fundamental, sendo também evidenciada em outras pesquisas ${ }^{(10,22)}$, corroborando com o presente estudo. Diversos estudos evidenciaram que a baixa escolaridade e renda fazem-se presentes nos pacientes com feridas crônicas ${ }^{(4,22)}$ o que interfere, muitas vezes, tanto na compreensão quanto na assimilação de cuidados à saúde, especialmente o cuidado com lesões, principalmente entre pacientes idosos com doenças crônicas, que precisam lidar com medicamentos, dietas e curativos ${ }^{(21-22)}$.

O principal tipo de ferida crônica identificada no estudo foi ferida operatória complicada (deiscente). Diferentemente dos resultados encontrados em estudo realizado com 202 pacientes internados com UD em seis hospitais de Portugal(20). A presença da infecção de sítio cirúrgico prolonga o tempo de internação para o seu tratamento, como observado no presente estudo. Além disso, o paciente internado está mais susceptível à aquisição de infecções e ao surgimento de novas lesões ${ }^{(22)}$.

Em relação aos domínios do FLQA-Wk, estudo que avaliou a QV utilizando o FLQA-W em 61 pacientes com diagnóstico de úlcera venosa na Alemanha identificou o pior resultado para o domínio Vida Diária $(3,61 \pm 0,93)$, e o melhor para o domínio Bem-Estar Psicológico $(2,76 \pm 1,08)^{(16)}$. Outro estudo que avaliou a QV utilizando o ShortForm Health Survey 36 (SF-36) em pacientes internados ${ }^{(20)}$ identificou que o escore dos componentes mentais foi superior ao escore dos componentes físicos, ambos resultados semelhantes ao encontrado neste estudo. Este resultado é interessante, uma vez que o comprometimento do domínio físico é esperado em pacientes com feridas crônicas, em vez disso, foi observado que o bem-estar psicológico obteve o melhor resultado e não estava tão comprometido como o esperado.

Quanto ao domínio Vida Social, este obteve a maior pontuação em relação aos escores dos outros domínios, resultado semelhante ao encontrado no estudo de validação do FLQA-W ${ }^{(12)}$, indicando maior interferência na vida social dos entrevistados, tal fato no presente estudo pode estar relacionado à impossibilidade dos indivíduos de exercerem suas atividades sociais, de lazer e de convívio familiar em decorrência do regime de hospitalização.

Foi encontrada associação significativa entre idade e os domínios Vida Diária e Vida Social, Satisfação e Escore Total do FLQA-Wk, resultado semelhante ao encontrado em estudo que identificou o pior resultado para o domínio Vida Diária( ${ }^{(15)}$. Com o aumento da idade, na medida em que esses pacientes demonstram alguma dependência para administrar suas atividades, sejam elas domiciliares, no lazer e nos meios social e familiar, podem ter sua autonomia prejudicada, tornando-se, dependentes de seus familiares e amigos ${ }^{(15,19,22)}$. Devido seu estado clínico fragilizado e localização da ferida, pacientes hospitalizados quase sempre dependem de outras pessoas, sejam elas cuidadores, familiares ou os próprios profissionais de saúde, para realização de suas atividades cotidianas como locomover-se, alimentar-se e ir ao banheiro, fato este que reflete na perda de sua autonomia e impacta negativamente em sua QV. 
Quanto à Satisfação, encontrou-se que quanto maior a idade, maior a satisfação do indivíduo em relação a sua saúde geral, tratamento e aparência da ferida crônica, resultado semelhante ao encontrado em estudo que evidenciou que pessoas mais jovens com feridas crônicas experimentaram uma QV significativamente pior quando comparada às pessoas mais velhas, uma vez que os mais jovens têm dificuldade em enfrentar o caráter crônico da doença e, portanto, não lidam da mesma forma que os mais velhos ${ }^{(23)}$.

Houve correlação entre a mobilidade e os domínios Vida Diária, Tratamento, o escore Total do FLQA-Wk e a EVA Satisfação com a Ferida, indicando forte relação entre pior QV quanto menor for a mobilidade. Estudos apontam que a presença ou história de feridas crônicas provoca grande impacto no funcionamento físico e na mobilidade ${ }^{(21,24)}$. De fato, a presença da ferida crônica afeta a percepção que o indivíduo tem sobre o seu bemestar físico e limita suas atividades de vida diária e laborais. A interferência na mobilidade e locomoção obriga os pacientes com feridas crônicas a reestruturarem as atividades do seu cotidiano e, em alguns casos, a sentirem-se dependentes de outros, dificultando também as relações sociais ${ }^{(12)}$.

O presente estudo encontrou associação significativa entre a área total da ferida com o domínios Vida Diária, diferentemente do resultado encontrado no estudo de validação do FLQA-W que mostrou baixa sensibilidade à mudança na área da ferida e a $\mathrm{QV}{ }^{(11)}$. Entretanto, é sabido que o tipo, tamanho e número de feridas impactam na QV, visto que pessoas com feridas crônicas apresentam grandes ou múltiplos curativos, fazendo com que se sintam envergonhadas ao se aproximar de outras pessoas, impactando diretamente no convívio social e familiar ${ }^{(21)}$.

As variáveis clínicas relacionadas a ferida, os sinais de infecção e sinais de cicatrização apresentaram associação significativa com o domínio Sintomas Físicos e a EVA Satisfação com a Ferida, respectivamente. Estudo qualitativo realizado na Austrália que investigou a QV de pessoas com feridas crônicas que se autotrataram identificou que a infecção da ferida era uma preocupação constante entre os indivíduos com feridas crônicas. A preocupação com a infeç̧ão era séria entre os participantes e ocorreu quando acreditavam estar em risco ou tiveram uma infecção, ou quando pensavam que os cuidados profissionais que receberam aumentaram o risco de infecção(25).

Apesar de no presente estudo ter sido identificado associação entre a área total da ferida e o domínio Vida Diária, pesquisa realizada no Brasil utilizando Índice de Qualidade de Vida de Ferrans e Powers-Wound (FPQLI-WV) encontrou que a cicatrização da ferida não correspondeu a um preditor de alterações na QV dos pacientes com feridas crônicas. Entretanto, conhecer a mínima mudança significativa na cicatrização de feridas é de grande importância para o paciente e serve como referência para o estabelecimento de metas terapêuticas com foco na QV do mesmo ${ }^{(10)}$.

O presente estudo traz como limitações o pequeno tamanho da amostra e o baixo nível de escolaridade dos entrevistados que dificultou a aplicação do questionário.

\section{CONCLUSÃO}

Os resultados mostraram que a QV dos portadores de feridas crônicas hospitalizados teve como média geral 3,15 e DP $\pm 0,63$, sendo que quanto maior o valor do escore, pior a QV. O domínio do FLQA-Wk com maior interferência na QV dos pacientes hospitalizados foi o domínio Vida Social e o de menor interferência, o domínio 
Bem-Estar Psicológico. Foi encontrada associação significativa entre maior idade, sexo masculino, mobilidade totalmente limitada, maior área total da ferida e sinais de infecção presentes com uma pior QV dos pacientes hospitalizados.

A avaliação da QV de pacientes internados com feridas crônicas torna-se relevante dadas as necessidades específicas decorrentes do regime de hospitalização e consequente impacto na QV dos pacientes. Espera-se que este estudo possa contribuir no planejamento e implementação de ações de enfermagem holísticas e individualizadas com foco nos aspectos biopsicossociais que colaborem para melhora da QV dos pacientes e sirva como subsídio para pesquisas futuras.

Estudos futuros devem ser conduzidos, visando à ampliação do tamanho da amostra, uso do instrumento FLQA-Wk e à compreensão da magnitude dos aspectos da QV de pacientes hospitalizados com feridas crônicas.

\section{REFERÊNCIAS}

1. Markova A, Mostow EN. US skin disease assessment: ulcer and wound care. Dermatol Clin [Internet]. 2012 [cited 2018 Dec 31];30(1):107-11, ix. Available from: https://doi.org/10.1016/j.det.2011.08.005.

2. Waidman MAP, Rocha SC, Correia JL, Brischiliari A, Marcon SS. O cotidiano do indivíduo com ferida crônica e sua saúde mental. Texto contexto - enferm. [Internet]. 2011 [cited 2018 Dec 31];20(4):691-9. Available from: http://dx.doi.org/10.1590/S0104$\underline{07072011000400007 .}$

3. Santos LRO, Avelino FVSD, Luz MHBA, Cavalcante TB, Silva JLM, Santos CAPS. Característica demográficas e clínicas de pacientes de unidade de terapia intensiva com úlcera por pressão. Rev enferm UFPE on line [Internet]. 2016 [cited 2018 Dec 31];10(supl.1):225-231. Available from: https://periodicos.ufpe.br/revistas/revistaenfermagem/article/viewFile/10944/12250. 4. Oliveira JC, Taquary SAS, Barbosa AM, Veronesi RJB. Pé diabético e amputações em pessoas internadas em hospital público: estudo transversal. ABCS Health Sciences [Internet]. 2016 [cited 2018 Dec 31];41(1):34-39. Available from:

https://doi.org/10.7322/abcshs.v41i1.843.

5. Oliveira PR, Carvalho VC, Felix CS, Paula AP, Santos-Silva J, Lima ALLM. The incidence and microbiological profile of surgical site infections following internal fixation of closed and open fractures. Rev Bras Ortop [Internet]. 2016 [cited 2018 Dec 31];51(4):396-9. Available from: https://doi.org/10.1016/j.rboe.2015.09.012.

6. Agência Nacional de Vigilância Sanitária. Critérios Diagnósticos de Infecções Relacionadas à Assistência à Saúde [Internet]. 2aed. Brasília: ANVISA, 2017 [cited 2018 Dec 31]. Available from: http://portal.anvisa.gov.br/documents/33852/3507912/Caderno+2++Critérios+Diagnósticos+de+Infecção+Relacionada+à+Assistência+à+Saúde/7485b45a-074f-4b34-8868-61f1e5724501.

7. Evangelista DG, Magalhães ERM, Moretão DIC, Stival MM, Lima LR. Impacto das feridas crônicas na qualidade de vida de usuários da estratégia de saúde da família. Revista de Enfermagem do Centro Oeste Mineiro [Internet]. 2012 [cited 2018 Dec 31];2(2):25463. Available from: http://www.seer.ufsj.edu.br/index.php/recom/article/view/15.

8. Salomé GM, Ferreira LM. Qualidade de vida em pacientes com úlcera venosa em terapia compressiva por bota de Unna. Rev Bras Cir Plástica [Internet]. 2012 [cited 2018 Dec 31];27(3):466-71. Available from: https://doi.org/10.1590/S1983-51752012000300024. 9. Santos LSF, Camacho ACLF, Oliveira BGRB, Nogueira GA, Joaquim FL. Influência da úlcera venosa na qualidade de vida dos pacientes: revisão integrativa. Rev enferm UFPE on line [Internet]. 2015 [cited 2018 Dec 31];9(supl. 3):7710-22. Available from: https://periodicos.ufpe.br/revistas/revistaenfermagem/article/view/10512.

10. Santos VLCG, Oliveira AS, Amaral AFS, Nishi ET, Junqueira JB, Kim SHP. Quality of life in patients with chronic wounds: magnitude of changes and predictive factors. Rev Esc Enferm USP [Internet]. 2017 [cited 2018 Dec 31]51:e03250. Available from: https://doi.org/10.1590/S1980-220X2016049603250.

11. Domingues EAR, Alexandre NMC, Silva JV. Cultural adaptation and validation of the Freiburg Life Quality Assessment - Wound Module to Brazilian Portuguese. Rev Lat Am Enfermagem [Internet]. 2016 [cited 2018 Dec 31];24:e2684. Available from:

https://doi.org/10.1590/1518-8345.0289.2684.

12. Augustin M, Herberger K, Rustenbach SJ, Schäfer I, Zschocke I, Blome C. Quality of life evaluation in wounds: validation of the Freiburg Life Quality Assessment-wound module, a disease-specific instrument. Int Wound J [Internet]. 2010 [cited 2018 Dec 31];7(6):493-501. Available from: https://doi.org/10.1111/j.1742-481X.2010.00732.x.

13. Purwins S, Herberger K, Debus ES, Rustenbach SJ, Pelzer $P$, Rabe E et al. Cost-of-illness of chronic leg ulcers in Germany. Int Wound J [Internet]. 2010 [cited 2018 Dec 31];7(2):97-102. Available from: https://doi.org/10.1111/i.1742-481X.2010.00660.x. 
14. Augustin M, Baade K, Heyer K, Price PE, Herberger K, Wild T et al. Quality-of-life evaluation in chronic wounds: comparative analysis of three disease-specific questionnaires. Int Wound J [Internet]. 2017 [cited 2018 Dec 31];14(6):1299-304. Available from: https://doi.org/10.1111/iwj.12803.

15. Miertová M, Dlugošová K, Ovšonková A, Čáp J. Chosen aspects of quality of life in patients with venous leg ulcers. Cent Eur J Nurs Midw [Internet]. 2016 [cited 2018 Dec 31];7(4):527-533. Available from: http://periodicals.osu.eu/cejnm/5 133 chosenaspects-of-quality-of-life-in-patients-with-venous-leg-ulcers.html.

16. Blome C, Baade K, Debus ES, Price P, Augustin M. The "Wound-QoL": a short questionnaire measuring quality of life in patients with chronic wounds based on three established disease-specific instruments. Wound Repair Regen [Internet]. 2014 [cited 2018 Dec 31];22(4):504-14. Available from: https://doi.org/10.1111/wrr.12193.

17. Almeida WA, Ferreira AM, Ivo ML, Rigotti MA, Gonçalves RQ, Pereira APS. Características sociodemográficas e clínicas e a qualidade de vida de pessoas com feridas: revisão integrativa. Rev enferm UFPE on line [Internet]. 2014 [cited 2018 Dec 31];8(12):4353-61. Available from: https://periodicos.ufpe.br/revistas/revistaenfermagem/article/view/10183.

18. Blay SL, Mari JDJ, Ramos LR, Ferraz MPT. Validity of a brazilian version of the mental status questionnaire as a screening test for dementia among elderly urban subjects. A pilot study. Int J Geriatr Psychiatry [Internet]. 1991 [cited 2018 Dec 31];6(11):779-85. Available from: https://doi.org/10.1002/gps.930061105.

19. Wachholz PA, Masuda PY, Nascimento DC, Taira CMH, Cleto NG. Quality of life profile and correlated factors in chronic leg ulcer patients in the mid-west of São Paulo State, Brazil. An Bras Dermatol [Internet]. 2014 [cited 2018 Dec 31];89(1):73-81. Available from: https://doi.org/10.1590/abd1806-4841.20142156.

20. Pedras S, Carvalho R, Pereira MG. Predictors of quality of life in patients with diabetic foot ulcer: the role of anxiety, depression, and functionality. J Health Psychol [Internet]. 2018 [cited 2018 Dec 31];23(11):1488-98. Available from:

https://doi.org/10.1177/1359105316656769.

21. Silva TG, Vasconcelos APL, Ramos EVC, Farias Neto JP. Avaliação da qualidade de vida de pacientes portadores de feridas crônicas atendidos no ambulatório de cicatrização do Hospital Universitário de Sergipe. Rev Bras Qual Vida [Internet]. 2017 [cited 2018 Dec 31];9(3):234-46. Available from: https://doi.org/10.3895/rbqv.v9n3.6704.

22. Almeida WA, Ferreira AM, Ivo ML, Rigotti MA, Barcelos LDS, Silva ALNV. Fatores associados à qualidade de vida de pessoas com feridas complexas crônicas. Revista de Pesquisa: Cuidado é Fundamental Online [Internet]. 2018 [cited 2018 Dec 31];10(1):9-16. Available from: https://doi.org/10.9789/2175-5361.2018.v10i1.9-16.

23. Kapp S, Santamaria N. The financial and quality-of-life cost to patients living with a chronic wound in the community. Int Wound J [Internet]. 2017 [cited 2018 Dec 31];14(6):1108-19. Available from: https://doi.org/10.1111/iwj.12767.

24. Nogueira GA, Oliveira BGRB, Santana RF, Cavalcanti ACD. Diagnósticos de enfermagem em pacientes com úlcera venosa crônica: estudo observaciona. Rev. Eletr. Enf [Internet]. 2015 [cited 2018 Dec 31];17(2):333-9. Available from: https://doi.org/10.5216/ree.v17i2.28782.

25. Kapp S, Miller C, Santamaria N. The quality of life of people who have chronic wounds and who self-treat. J Clin Nurs [Internet]. 2018 [cited 2018 Dec 31];27(1-2):182-92. Available from: https://doi.org/10.1111/jocn.13870. 